\section{Comment: Is memristor a dynamic element?}

R. Riaza

In [1] the authors present a charge/flux formulation of the equations of memristive circuits which seemingly show that the memristor should not be considered as a dynamic circuit element. We show in this Letter that the authors' approach implicitly reduces the dynamic analysis to a certain subset of the state space in a way such that the dynamic contribution of memristors is hidden. This reduction might entail a substantial loss of information, regarding e.g. the local stability properties of the circuit. Two examples illustrate this. We conclude that the memristor, even with its unconventional features, must be considered as a dynamic element.

Introduction: Memristors $[2,8]$ are known to display unconventional dynamical features; notably, equilibrium points of memristive circuits are not isolated [5, 7]. Equilibria in a nonlinear circuit including $m$ memristors lie on $m$-dimensional sets. However, this does not mean that memristors do not contribute to the state space dimension of the circuit (that is, the dimension of the space where solutions lie or, equivalently, the number of initial conditions which can be freely assigned). On the contrary, this property of memristors is responsible for subtle dynamical properties which require a delicate analysis.

In [1], the authors propose a charge/flux formulation of the equations of memristive circuits which seemingly prove that the memristor is not a dynamic element, namely, that it does not actually contribute to the state dimension of the circuit. We show below that the approach of [1] implicitly reduces the analysis to a lower dimensional (so-called invariant) manifold of the actual state space, and that the number of disregarded dimensions (or codimension) equals the number of memristors. We apply these results to the canonical Chua's memristor oscillator considered in [1], showing that the results there are restricted to a three-dimensional subset of the full space space, which is four-dimensional. We also discuss a simpler circuit with just two devices for a better understanding of this matter.

Charge/flux formulation of memristive circuit equations: As in [1], we consider a lumped circuit with $k$ branches, composed of $r$ linear resistors, $c$ linear capacitors, $l$ linear inductors and $m$ memristors, with $k=r+c+$ $l+m$. All devices are assumed to be time-invariant and their constitutive relations will be written as $v_{r}=R i_{r}$ (resistors), $q_{c}=C v_{c}$ (capacitors), $\varphi_{l}=L i_{l}$ (inductors) and

$$
\varphi_{m}=\phi\left(q_{m}\right)
$$

(memristors), with $\phi: \mathbb{R}^{m} \rightarrow \mathbb{R}^{m}$; note that $R, C$ and $L$ are resistance, capacitance and inductance matrices. For the sake of simplicity we ignore voltage and current sources but they can be accommodated in the discussion in a straightforward manner; the same happens with fluxcontrolled memristors.

Time-integration of Kirchhoff laws makes it possible to reformulate them in terms of charges $q$ and fluxes $\varphi$ as

$$
\begin{aligned}
& A q(t)=A q(0) \\
& B \varphi(t)=B \varphi(0) .
\end{aligned}
$$

Here $A$ and $B$ are reduced incidence and loop matrices (see e.g. [3, 6]) and $q, \varphi$ join together all charges/fluxes; that is, $q=\left(q_{r}, q_{c}, q_{l}, q_{m}\right), \varphi=$ $\left(\varphi_{r}, \varphi_{c}, \varphi_{l}, \varphi_{m}\right)$. It is well known in circuit theory that Kirchhoff laws specify $k$ linearly independent relations between the circuit variables.

To get a full description of the circuit dynamics in terms of charges and fluxes, we also need to reformulate the equations for resistors, capacitors and inductors. By time-integration of $v_{r}=R i_{r}$, the charge/flux equations

This is the author's copy of the paper published in Electronics Letters, Volume 50, Issue 19, 11 September 2014, pp. 1342-1344. $\mathrm{http} / /$ digital-library.theiet.org/content/journals/10.1049/el.2014.1553 for resistors should read as

$$
\varphi_{r}(t)-R q_{r}(t)=\varphi_{r}(0)-R q_{r}(0)
$$

whereas the equations for capacitors and inductors are

$$
\begin{aligned}
C \varphi_{c}^{\prime}(t) & =q_{c}(t) \\
L q_{l}^{\prime}(t) & =\varphi_{l}(t) .
\end{aligned}
$$

The system defined by (1), (2), (3) and (4) specifies the charge/flux dynamics of the circuit. Note that this model involves a total amount of $2 k=2(r+c+l+m)$ variables.

In [1], the authors implicitly assume that the right-hand sides of (2) and (3) do vanish. This key assumption implies that both equations impose $k+r$ linear restrictions among the circuit variables. Since (1) imposes $m$ additional restrictions, there is a total amount of $k+r+m$ restrictions among the $2 k$ variables, yielding a state dimension or dynamical degree of freedom of $2 k-(k+r+m)=k-r-m=c+l$. Equivalently, in terms of initial values, the aforementioned vanishing assumption on the initial conditions implies that only $c+l$ initial values can be freely assigned. Under this assumption, the state dimension is indeed defined by the total number of capacitors and inductors, and it seems that the memristors do not contribute to this dimension.

This contradicts what one would expect from the conventional formulation, which is defined by the equations $C v_{c}^{\prime}=i_{c}, L i_{l}^{\prime}=v_{l}$, $q_{m}^{\prime}=i_{m} \quad v_{r}=R i_{r}, v_{m}=M\left(q_{m}\right) i_{m}$ (where $M\left(q_{m}\right)=\phi^{\prime}\left(q_{m}\right)$ is the memristance), together with Kirchhoff laws $A i=0, B v=0$. Here $2 k+m$ variables are involved (all voltages and currents besides the memristors charges $q_{m}$ ), and there are $r+m+k$ restrictions, yielding a state dimension of $c+l+m$; notice the contribution of memristors to this dimension.

As illustrated by the examples below, the conventional formulation is of course correct, and the absence of memristors in the state dimension as computed in [1] is a consequence of the fact that more initial conditions than necessary are imposed by the vanishing assumption indicated above.

Chua's memristor oscillator: Figure 1 displays the canonical Chua's oscillator with a flux-controlled memristor [4].

$\mathrm{L}$

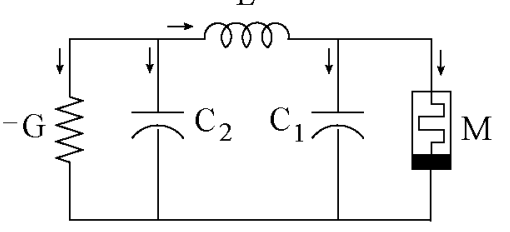

Fig. 1. Canonical Chua's memristor oscillator

We will denote by $\left(q_{c_{1}}, q_{c_{2}}, q_{l}, q_{m}, q_{r}\right)$ and $\left(\varphi_{c_{1}}, \varphi_{c_{2}}, \varphi_{l}, \varphi_{m}, \varphi_{r}\right)$ the charges and the fluxes at the capacitors $C_{1}$ and $C_{2}$, the inductor, the memristor and the resistor. The memristor is a flux-controlled one, and its characteristic $q_{m}=\zeta\left(\varphi_{m}\right)$ is assumed to be differentiable, with $W\left(\varphi_{m}\right)=$ $\zeta^{\prime}\left(\varphi_{m}\right)$. It is straightforward to write the circuit equations in terms of the capacitor charges and the fluxes at the inductor and the memristor (cf. eq. (38) in [4]); these are

$$
\begin{aligned}
q_{c_{1}}^{\prime} & =\varphi_{l} / L-W\left(\varphi_{m}\right) q_{c_{1}} / C_{1} \\
q_{c_{2}}^{\prime} & =-\varphi_{l} / L+G q_{c_{2}} / C_{2} \\
\varphi_{l}^{\prime} & =-q_{c_{1}} / C_{1}+q_{c_{2}} / C_{2} \\
\varphi_{m}^{\prime} & =q_{c_{1}} / C_{1} .
\end{aligned}
$$

By using, as in [1], equations (2) and (3) with a vanishing right-hand side, one gets the restrictions $q_{r}+q_{c_{2}}+q_{l}=0, q_{l}-q_{c_{1}}-q_{m}=0, \varphi_{T}=$ $\varphi_{c_{2}}, \varphi_{c_{2}}-\varphi_{c_{1}}-\varphi_{l}=0, \varphi_{c_{1}}=\varphi_{m}$ and $q_{r}+G \varphi_{r}=0$. Together with $q_{m}=\zeta\left(\varphi_{m}\right)$, these seven restrictions allow one to express the six variables $q_{l}, q_{m}, q_{r}, \varphi_{c_{1}}, \varphi_{c_{2}}$ and $\varphi_{r}$ in terms of the four variables involved in (5); 
only one restriction remains and this can be easily seen to read as

$$
q_{c_{1}}+q_{c_{2}}+\zeta\left(\varphi_{m}\right)-G\left(\varphi_{m}+\varphi_{l}\right)=0 .
$$

This equation defines a three-dimensional invariant manifold for the flow defined by (5). This means that, provided that an initial point satisfies (6), the trajectory emanating from it remains in the manifold defined by (6). This property can be shown to hold by checking that the time derivative of the left-hand side of (6) vanishes:

$$
\begin{aligned}
q_{c_{1}}^{\prime} & +q_{c_{2}}^{\prime}+\left(\zeta\left(\varphi_{m}\right)\right)^{\prime}-G\left(\varphi_{m}^{\prime}+\varphi_{l}^{\prime}\right)= \\
& =i_{c_{1}}+i_{c_{2}}+W\left(\varphi_{m}\right) v_{m}-G v_{m}-G v_{l}= \\
& =i_{c_{1}}+i_{c_{2}}+i_{m}+i_{r}=0
\end{aligned}
$$

where the last identity owes to the fact that the four vertical branches in Fig. 1 define a cutset and hence the total current flowing through it is zero.

Moreover, by restricting the system (5) to the manifold defined by (6) one gets the three-dimensional model discussed in [1]. Indeed, by writing $q_{c_{1}}$ in terms of $q_{c_{2}}, \varphi_{l}$ and $\varphi_{m}$ via (6), equations (5b), (5c) and (5d) yield

$$
\begin{aligned}
q_{c_{2}}^{\prime} & =-\varphi_{l} / L+G q_{c_{2}} / C_{2} \\
\varphi_{l}^{\prime} & =\left(q_{c_{2}}+\zeta\left(\varphi_{m}\right)-G\left(\varphi_{m}+\varphi_{l}\right)\right) / C_{1}+q_{c_{2}} / C_{2} \\
\varphi_{m}^{\prime} & =-\left(q_{c_{2}}+\zeta\left(\varphi_{m}\right)-G\left(\varphi_{m}+\varphi_{l}\right)\right) / C_{1} .
\end{aligned}
$$

By means of the linear coordinate change defined by the identities $x=\varphi_{m}$, $y=\varphi_{m}+\varphi_{l}, z=-q_{c_{2}}+G\left(\varphi_{m}+\varphi_{l}\right)$ and setting $1 / C_{1}=\alpha, 1 / C_{2}=\beta$, $G / C_{2}=\gamma, L=1$, as in [1], one easily recasts (7) as

$$
\begin{aligned}
& x^{\prime}=\alpha(z-\zeta(x)) \\
& y^{\prime}=\gamma y-\beta z \\
& z^{\prime}=-x+y
\end{aligned}
$$

which is exactly eq. (13) in [1]. This shows that the three-dimensional system derived in [1] only describes the flow of Chua's memristor oscillator on the invariant manifold defined by (6); the whole flow is fourdimensional and is fully described by (5). The distinction is important because the flow on other invariant manifolds may exhibit a completely different behavior; this is illustrated below by means of a simpler example.

A two-device example: The results discussed above can be further illustrated by a simpler circuit example just defined by the connection of a linear inductor and a charge-controlled memristor, as depicted in Figure 2.

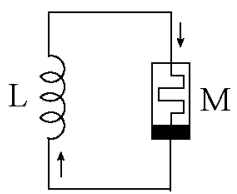

Fig. 2. ML circuit

Let $\varphi_{m}=\phi\left(q_{m}\right)$ be the characteristic of the memristor and write $M\left(q_{m}\right)=\phi^{\prime}\left(q_{m}\right)$. It is straightforward to write the equations of such circuit, which in terms of $q_{m}$ and the inductor current $i_{l}$ read as

$$
\begin{aligned}
L i_{l}^{\prime} & =-M\left(q_{m}\right) i_{l} \\
q_{m}^{\prime} & =i_{l} .
\end{aligned}
$$

Note that $i_{l}=0$ defines a curve of (non-isolated) equilibrium points. For the sake of simplicity let as fix $L=1$ and assume that $\phi\left(q_{m}\right)=q_{m}^{2} / 2$, so that $M\left(q_{m}\right)=q_{m}$. By recasting (9) in terms of $\frac{i_{l}^{\prime}}{q_{m}^{\prime}}=\frac{d i_{l}}{d q_{m}}$, it is not difficult to check that the trajectories lie on curves of the form

$$
i_{l}=-q_{m}^{2} / 2+k
$$

for any real constant $k$. The actual trajectory behavior is displayed in Figure 3. Equilibria, displayed in a continuous straight line, are located on the (vertical) $q_{m}$-axis.

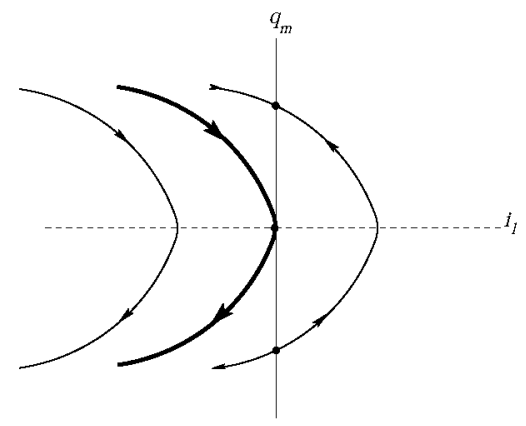

Fig. 3. Possible trajectory behavior in the ML circuit

For $k<0$, trajectories do not reach the equilibrium line; these are located on the left of the boldface curve in the Figure. This boldface curve corresponds to the case $k=0$ and includes an equilibrium at the origin. The trajectory on this curve with $q_{m}>0$ approaches asymptotically the equilibrium point located at the origin, whereas the trajectory with $q_{m}<0$ leaves asymptotically the equilibrium and escapes to infinity.

Finally, curves on the right of the boldface one comprise two equilibrium points in the $q_{m}$ axis. Trajectories leave and/or approach asymptotically an equilibrium as indicated by the arrows in Figure 3. One can check that equilibria defined by $i_{l}=0, q_{m}>0$ are stable, whereas those with $i_{l}=0, q_{m}<0$ are unstable. This indicates that the origin behaves as a bifurcating point.

By contrast, following the approach of [1] we would get the relations $q_{l}=q_{m}, \varphi_{l}+\varphi_{m}=0, \varphi_{m}=\phi\left(q_{m}\right)$ and a single differential equation

$$
L q_{l}^{\prime}=-\phi\left(q_{l}\right)
$$

With $L=1$ and $\phi(q)=q^{2} / 2$, as above, one easily gets $q_{l}=\frac{2 q_{l}(0)}{2+t q_{l}(0)}$. By using $q_{m}=q_{l}$ and $i_{l}=q_{l}^{\prime}$, we obtain that trajectories of this model are forced to lie on the curve defined by

$$
i_{l}=-q_{m}^{2} / 2,
$$

which corresponds to $k=0$ in (10) and to the boldface curve in Fig. 3, which includes the equilibrium at the origin. This is only one invariant curve in the whole phase portrait, which is two-dimensional. It would not be accurate to conclude that this curve describes the whole dynamics of the circuit since, as indicated above, very different trajectory behaviors do happen within other invariant curves. Specifically, other curves may accommodate no equilibria or, on the contrary, two equilibrium points.

Concluding remarks: The results above show that the charge/flux approach of [1] provides a description of the dynamics of memristive circuits in lower dimensional invariant manifolds of the actual state space, with the number of disregarded dimensions given by the number of memristors. When considering the full dynamic flow of a circuit with memristors, these are responsible for unconventional phenomena such as the existence of manifolds of non-isolated equilibria. The local dynamical properties of the circuit may change as we move along such manifolds. Therefore the memristor must be considered as a dynamic element which actually plays a key role in the qualitative behavior of the circuit.

Acknowledgment: Research supported by Project MTM2010-15102 of Ministerio de Ciencia e Innovación, Spain.

Ricardo Riaza (Depto. Matemática Aplicada TTI, ETSI Telecomunicación, Universidad Politécnica de Madrid, Spain)

E-mail: ricardo.riaza@upm.es

\section{References}

1 B. C. Bao, Z. Liu and H. Leung, Is memristor a dynamic element?, Electronics Letters 49 (2013) 1523-1525.

2 L. O. Chua, Memristor - The missing circuit element, IEEE Trans. Circuit Theory 18 (1971) 507-519.

3 L. O. Chua, C. A. Desoer and E. S. Kuh, Linear and Nonlinear Circuits, McGraw-Hill, 1987. 
4 M. Itoh and L. O. Chua, Memristor oscillators, Intl. J. Bifur. Chaos 18 (2008) 3183-3206.

5 M. Messias, C. Nespoli and V. A. Botta, Hopf bifurcation from lines of equilibria without parameters in memristors oscillators, Int1. J. Bifur. Chaos 20 (2010) 437-450.

6 R. Riaza, Differential-Algebraic Systems, World Scientific, 2008.

7 R. Riaza, Manifolds of equilibria and bifurcations without parameters in memristive circuits, SIAM J. Appl. Math. 72 (2012) 877-896.

8 D. B. Strukov, G. S. Snider, D. R. Stewart and R. S. Williams, The missing memristor found, Nature $\mathbf{4 5 3}$ (2008) 80-83. 